\title{
Systems-based approaches toward wound healing
}

\author{
Adrian Buganza Tepole' and Ellen Kuhl1,2
}

Wound healing in the pediatric patient is of utmost clinical and social importance because hypertrophic scarring can have aesthetic and psychological sequelae, from early childhood to late adolescence. Wound healing is a well-orchestrated reparative response affecting the damaged tissue at the cellular, tissue, organ, and system scales. Although tremendous progress has been made toward understanding wound healing at the individual temporal and spatial scales, its effects across the scales remain severely understudied and poorly understood. Here, we discuss the critical need for systems-based computational modeling of wound healing across the scales, from short-term to long-term and from small to large. We illustrate the state of the art in systems modeling by means of three key signaling mechanisms: oxygen tension-regulating angiogenesis and revascularization; transforming growth factor- $\beta$ (TGF- $\beta$ ) kinetics controlling collagen deposition; and mechanical stretch stimulating cellular mitosis and extracellular matrix (ECM) remodeling. The complex network of biochemical and biomechanical signaling mechanisms and the multiscale character of the healing process make systems modeling an integral tool in exploring personalized strategies for wound repair. A better mechanistic understanding of wound healing in the pediatric patient could open new avenues in treating children with skin disorders such as birth defects, skin cancer, wounds, and burn injuries.

D ermal wound healing in the pediatric patient is a symphony of events precisely synchronized to repair the damaged tissue, restore its protective barrier function, and safely return it to its homeostatic equilibrium state (1). Although the underlying processes, cell-matrix interaction, cell-cell cross-talk, and cellular mechanotransduction, involve a complex cascade of events, dermal wound healing is robust and rarely diverges to malignant transformation (2). Yet it is not always perfect. Prenatal skin usually heals smoothly to seamlessly restore the state before injury (3), whereas postnatal skin is incapable of healing wounds tracelessly, leaving scar behind. Postnatal skin can easily restore its protective barrier function; however, the resulting scar rarely has the same microstructure, collagen content, and mechanical properties as the native tissue (4). In extreme cases, pronounced fibrotic activity might even initiate hypertrophic scaring, characterized by an excessive collagen deposition (5). In the pediatric patient, excessive scarring has consequences throughout early childhood and adolescence and can lead to low self-esteem or even stigmatization (6). The prevalence of hypertrophic scarring in the pediatric population is overwhelming: of the total cases of burn scars and keloids, $70 \%$ occur in children (7).

The underlying mechanisms of scar formation are now better understood than ever before, and we have made tremendous progress toward improving and accelerating healing mechanisms (8). We have come to appreciate that the healing process spans various temporal and spatial scales and is affected by both chemical and mechanical cues. However, even with the detailed insight that traditional approaches have provided on the individual scales, the behavior of the system as a whole remains elusive. Computational modeling is increasingly recognized as a powerful tool to provide insight into the dynamics of wound healing and the interaction of biochemical and biomechanical phenomena across the different scales (9).

Fortunately, dermal wound healing, like all inflammationbased processes in the human body, is based on various redundant signals and cross-talk between different signaling networks (10). Although redundancy is hugely beneficial for the biological system itself, it complicates the overall understanding of the healing process: even if individual elements of the signaling network are well understood in isolation, the coupling of these elements is hugely complex, and it is virtually impossible to gain basic insights based on sparse experimental data. Computational systems biology is of acknowledged importance in advancing our holistic understanding of pediatric wound healing: in the short term, computational systems biology allows us to systematically explore controlled what-if scenarios and virtually probe various hypotheses to better understand the healing process as a whole (11); in the long term, given the incredible variability of healing responses between different individuals, computational systems biology is an integral ingredient to shape the future of personalized medicine (12).

\section{TEMPORAL SPECTRUM OF WOUND HEALING}

From the moment of injury until the tissue reaches its final configuration in the form of a mature scar, months or even years may elapse. However, the protective function of skin has to resume immediately to avoid dehydration, infection, and loss of tissue integrity. Accordingly, the initial phase of healing takes only a few minutes. The process of wound healing is commonly divided into four overlapping phases: hemostasis, 
inflammation, proliferation, and remodeling (see Figure 1, rows 1 through 4 ).

\section{During Hemostasis, the Injured Region Fills With a Platelet-Rich Fibrin Clot}

Hemostasis occurs in the order of minutes. During hemostasis, the wounded space rapidly fills with a clot to stop blood loss and to re-establish a barrier from the outside world. In the later phases of healing, this clot will serve as a temporary matrix for the cells that migrate toward the wound to reconstruct the dermal tissue (Figure 1; 1c) (13). The clot is composed mainly of fibrin fibers and platelets. The cytoplasm of the platelets carries $\alpha$-granules from which a cocktail of growth factors and cytokinesis is released when the cells degranulate (Figure 1; 1b) (14). Proinflammatory signals from the platelets are directly reflected in molecular changes in the endothelial cells of the blood vessels near the injury site. A chemotactic response attracts leukocytes such as neutrophils and monocytes (Figure 1; 1a), which sense these molecular changes and adhere to the endothelial cells. The ongoing chemotactic pathway and the interaction between leukocytes and endothelial cells lead to the capture and transmigration of the neutrophils and monocytes in a process called diapedesis (15).

\section{During Inflammation, Leukocytes Establish Chemotactic Gradients}

Neutrophil diapedesis marks the beginning of the inflammatory phase. Neutrophil transmigration also occurs very rapidly; in fact, the first neutrophils reach the wound within a few minutes, but they keep being recruited for the next 2 or $3 \mathrm{~d}$. The primary function of neutrophils is to dispose of pathogens entrapped in the clot at the moment of injury. Their secondary function is to amplify the proinflammatory indicators. Monocytes arrive at the wound within $2 \mathrm{~d}$ of the initial insult and differentiate into

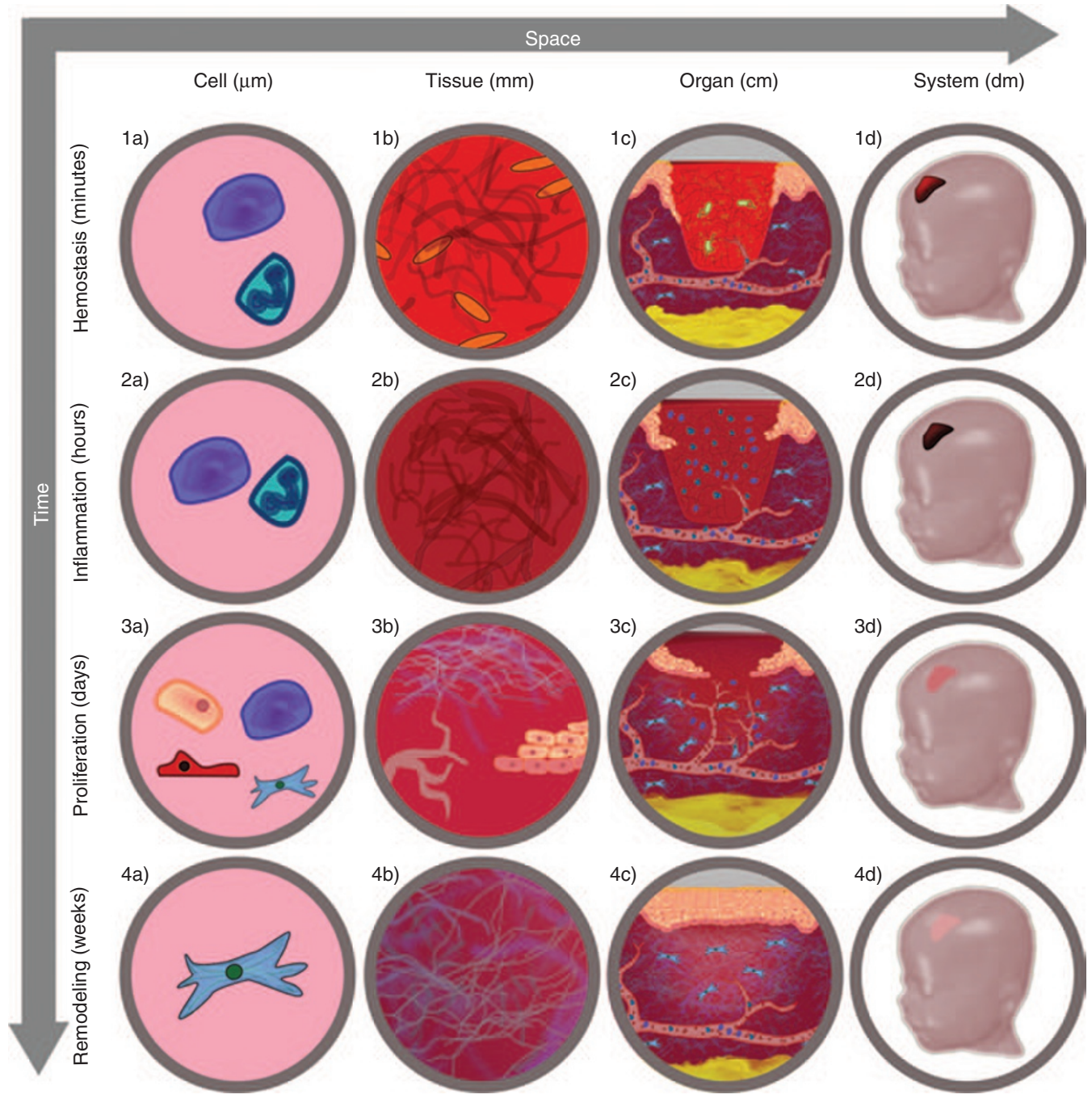

Figure 1. Spatiotemporal spectrum of wound healing. Wound healing is a hierarchically orchestrated process that spans interacting phenomena from minutes to months, from the cellular to the system level. Temporally, homeostasis takes place within the first minutes (row 1 ), inflammation within hours (row 2), proliferation within days (row 3), and remodeling within weeks to months (row 4). Spatially, the cellular level is associated with length scales in the order of micrometers (column a), the tissue level with millimeters (column b), the organ level with centimeters (column c), and the system level with decimeters (column d). 
macrophages (Figure 1; 2a). The role of monocytes is twofold: they phagocytose the remaining matrix debris, pathogens, and neutrophils, and they produce soluble chemical mediators such as transforming growth factor- $\beta$ (TGF- $\beta$ ) and vascular endothelial growth factor. Both TGF- $\beta$ and vascular endothelial growth factor are crucial to guiding the recruitment of fibroblasts and endothelial cells later in the healing process (Figure 1; 2c).

\section{During Proliferation, Fibroblasts Deposit Collagen and New Vasculature Forms}

During the proliferative phase, two processes occur simultaneously: re-epithelialization and replacement of the fibrin clot by granulation tissue (Figure 1; 3c). These steps in the repair process continue throughout $2-3 \mathrm{wk}$. In this phase, the sharp chemoattractant gradients generated through the inflammatory phase attract different cell types to manufacture new tissue (Figure 1; 3a). At the epidermal level, keratinocytes in the wound edge dissolve their adhesions to the basal lamina in order to crawl and reproduce on top of the fibrin matrix, rebuilding the epidermis over the injured region (Figure 1; $3 \mathbf{b}$ ). On the dermal level, fibroblasts are in charge of depositing collagen, the main load-bearing constituent of skin (Figure 1; 3b). The metabolic needs resulting from increased cellular proliferation and migration require the formation of a vast network of capillary tubes to provide nutrients and oxygen. Endothelial cells rapidly forma new vasculature in a process referred to as angiogenesis (Figure 1; 3b). Toward the end of this phase, some fibroblasts transform into myofibroblasts, which actively pull on the wound edges to contract the injured tissue.

\section{During Remodeling, Fibroblasts Gradually Restore Skin Integrity and Homeostasis}

Once the fibrin matrix has been replaced by granulation tissue made of fibroblasts, the remaining macrophages, and a vascular network, the remodeling phase begins. At this point, the wound is fully closed, yet the tissue has very poor quality. It consists primarily of thick, aligned collagen bundles, instead of the interwoven collagen networks found in native skin (Figure 1; $4 b$ ). Dynamic changes continue, but on a much slower time scale. Remodeling can go on for months or even years. Finally, the vascular network retracts and most of the cells undergo apoptosis or migrate out of the affected region. The remaining fibroblasts (Figure 1; 4a) gradually restore skin integrity and mechanical homeostasis (Figure 1; 4c). However, unlike prenatal skin, postnatal skin usually never fully recovers its original native state.

\section{SPATIAL SPECTRUM OF WOUND HEALING}

Similar to virtually all biological phenomena, in wound healing, temporally and spatially interacting events at the molecular, subcellular, cellular, tissue, and organ levels ultimately converge to a well-defined system-level response (16) (see Figure 1, columns a through $\mathrm{d}$ ). This macroscopic response is inherently rooted in the hierarchical structure, which is established through a precisely defined biological organization (17). Although it is in principle possible to zoom in to the subcellular or even molecular scales, the cellular level is the preferred starting point in the context of wound healing modeling, mainly because cells are the smallest autonomous building blocks in the biological hierarchy (18). One level above in the organization, at the tissue level, we find interacting cell aggregates embedded in the extracellular matrix (ECM). The next larger scale corresponds to the organ level, the level of the wound itself. In principle, we could even contemplate higher levels of organization, for example, entire organ systems. Here, however, we do not consider larger spatial scales, because all relevant phenomena take place within the local wound environment.

\section{At the Cellular Level, Chemomechanical Stimuli Guide Cellular Decision Making}

The smallest scale we explore here is the cellular level, a scale in the order of $10 \mu \mathrm{m}$. At this scale, four cell types are critical to wound healing: endothelial cells, fibroblasts, macrophages, and keratinocytes (Figure 1; 1a-4a). For each cell type, there are specific regulatory mechanisms that are key to a successful repair process. The most important control mechanism is chemotaxis, the migration of cells in the direction of increasing chemical gradients (19). Endothelial cells, fibroblasts, and leukocytes migrate into the wound site attracted by the strong concentration of different growth factors and other chemical mediators released during the inflammatory phase (20-22). Chemotaxis in eukaryotic cells is complex and, unlike for prokaryotic systems, there are no established models to predict how eukaryotic cells migrate as a function of chemical gradients. Prokaryotic cell migration resembles a biased random walk, which can be easily represented by simple mathematical models. Eukaryotic cells, however, move in continuous paths with smooth turns toward ascending signal concentrations but display an overall stochastic behavior (23-25).

In addition to chemical cues, mechanical cues play a significant role in wound healing $(26,27)$. The ultimate goal of skin repair is to re-establish the mechanical load-bearing capacity to restore the homeostatic equilibrium state. Key to this restoration is the translation of mechanical signals into chemical reactions, a process known as mechanotransduction, which is the landmark characteristic of fibroblasts in the repair sequence (Figure $1 ; 4 a$ ) (28). Fibroblasts anchor to the ECM through focal adhesions. These adhesion sites are linked to stretch-activated ion channels across the cell membrane and to the cytoskeleton inside the cell. Altering the mechanical scenario of the ECM directly creates a flow of charged ions via these ion channels and indirectly governs cellular behavior through perturbed cytoskeletal dynamics (29). Fibroblasts respond to mechanical changes in the ECM in several ways: by depositing collagen, decreasing apoptosis rates, increasing inflammatory signals, and transforming into myofibroblasts to actively pull on the wound edges (30-32).

For endothelial cells and keratinocytes (Figure 1; 3a), cellcell and cell-matrix adhesions are of primary importance throughout the entire repair process. Keratinocytes critically depend on cell-cell and cell-matrix cross-talk to define their position in the epidermal lattice. The polarization of keratinocytes directs lateral migration and proliferation during reepithelialization (33). Endothelial cells also depend on these types of interaction during angiogenesis (34). Some cells in the tip of sprouting vessels are activated and protrude filopodial 
extensions, which actively interact with their immediate microenvironment to guide angiogenesis. The remaining endothelial cells support the leading tip by maintaining the connectivity with the parent vessels and by helping in the maturation of the newly formed capillary tubes (35).

\section{At the Tissue Level, Chemomechanical Fields Coordinate Cellular Organization}

On the next level of the hierarchy, we are interested in the bulk mechanical properties of the ECM, the diffusion of growth factors and the overall behavior of cellular aggregates (Figure $1 ; 1 b-4 b$ ). The properties of the ECM and the diffusion of growth factors are crucial at this scale, because they contribute a key aspect of the healing process: they pass information across distances larger than the characteristic cell size, coordinating the action of multiple cells without using direct cell-cell communication. Soluble mediators diffuse chemical cues, and the ECM transmits mechanical cues across the tissue (Figure $\mathbf{1} ; \mathbf{1 b}$ and $\mathbf{2 b}$ ). To characterize chemical diffusion in an isotropic medium, only a single constant needs to be specified. To characterize mechanical signaling of the ECM, however, we can select from a huge variety of constitutive models, and two or even more constants need to be determined (Figure 1; 4b) (36-38).

Two other phenomena that become important at the tissue level are pattern formation and collective cell migration. An illustrative example is re-epithelialization, associated with the formation of an advancing front that has distinct collective features entirely different from those of the individual cellular response. The leading edge of keratinocytes that invade the wound adopts a wave propagation profile, which can be characterized using Fisher's equation (Figure 1; 3b) (39). Another even more intriguing proof of complex organization is angiogenesis, a highly coordinated process that cannot be understood solely from the study of a single cell. During angiogenesis, a new vasculature forms from existing blood vessels that surround the injured region (40). This process consists of combined tip chemotactic diffusion and sprouting to create a new network of capillary tubes (Figure 1; 3b) (41).

\section{At the Organ Level, the Chemomechanical Environment Controls Wound Healing}

The final level of interest in wound healing is the spatial region that contains the injured skin and its immediately surrounding healthy tissue (Figure 1; 1c-4c). At this level, we witness the healing capacities of skin as the result of a perfectly organized interaction of all phenomena described above: from microstructural decisions of the individual cells, via mesostructural properties of the cells embedded in the ECM, to macrostructural reaction-diffusion systems of the soluble mediators and the patterning capabilities of cellular aggregates.

\section{OVERVIEW OF COMPUTATIONAL MODELING FOR SYSTEMS BIOLOGY}

Mathematical representations can foster our understanding of biological systems. An abstraction of a biological system consists of characteristic variables, such as the concentration of a chemical substance or the cell density in a region of tissue, and the interaction rules between the different components of the system. Mathematical models are representations of the real world and, as such, they can be created in many different ways depending on the features of the problem itself, on the information at hand, and on the mathematical tools preferred by the modeler. Creating a mathematical model is only the first step of a simulation process. The second step consists of converting the mathematical model into a computational model that allows solving complex patient-specific problems.

\section{Mathematical Models Are Sets of Equations That Characterize the Dynamics of a Biological System}

In the context of wound healing, there are two conceptually different mathematical approaches to representing a biological system: continuum models and discrete models. In the physical world, substances can vary smoothly over a given domain. In this case, we represent them through a function called a continuous field. Continuous fields are the basic representation of continuum models. Typical continuous fields in wound healing are concentrations of chemical mediators such as TGF- $\beta$. Some components of the system may be large enough to be considered as discrete entities. To model individual entities, we typically use discrete models that represent the individual components explicitly and characterize their interaction through simple mathematical equations. Typical discrete entities in wound healing are cell populations such as densities of fibroblasts and macrophages. The choice between continuum and discrete models strongly depends the particular scale of interest. Components that can be considered as discrete entities at the molecular and cellular level become averaged out at the tissue and organ level, at which they can be approximated as continuous fields. In general, the scale of interest will determine which modeling approach is more appropriate.

In systems biology, different types of interacting components have to be represented through the mathematical model, either as continuous fields or as discrete entities. A multifield model is one that consists of several interacting fields. Typical multifield phenomena in wound healing models are various interacting chemical signaling pathways, such as the TGF- $\beta$ and vascular endothelial growth factor pathways. In some cases, it might be advantageous to represent some variables as continuous fields whereas others might be represented as discrete entities. Accordingly, there are three basic types of representations: purely continuum, purely discrete, and hybrid continuum/ discrete. In addition, a mathematical model for each of these representations can span various spatial scales, from the cellular level, via the tissue and organ levels, to the system level. We characterize these types of models as multiscale models.

From a modeling point of view, biological systems consist of three basic ingredients: chemical substances, cell populations, and mechanical factors. Chemical substances are generally treated as continuous fields. Cell populations can either be modeled individually or as fields, depending on the scale of interest. Here, we use the notion of biological field for fields that represent cell populations. Mechanical factors include forces and deformations and are almost always treated as continuous fields. 
In continuum models, the evolution of different fields in space and time is governed by partial differential equations. A partial differential equation establishes (i) how a field interacts with other fields through coupling terms; (ii) how a field evolves in time; and (iii) how a field varies in space over the domain of interest. One partial differential equation is needed for each field. Continuum models represented through partial differential equations are an elegant and compact way to characterize spatial variations of chemical concentrations, mechanical strains, or mechanical stresses.

In discrete models, the behavior of each entity is governed by one or more ordinary differential equations. An ordinary differential equation establishes (i) how a characteristic variable of a single entity interacts with other variables through coupling terms; and (ii) how a characteristic variable evolves in time.

\section{Computational Models Are Algorithmic Realizations of a Mathematical Model}

In pediatric wound healing, the mathematical model is typically too complex to be solved analytically. Through a process called discretization, we can transfer the mathematical model, the set of equations, into a computational model, an algorithmic solution of these equations, that can easily simulate phenomena of various degrees of complexity.

Continuum models are typically represented through coupled partial differential equations. To solve the underlying set of equations, it is necessary to subdivide or discretize both space and time. Time is almost always discretized into small intervals by means of the finite difference methods. Discretizing space can be preformed in several ways: the simplest approach to represent the domain of interest is a regular mesh, as is used in the finite difference method and the finite volume method. To represent arbitrarily shaped domains such as particular parts of the body, the preferred approach is the use the finite element method. This method approximates the domain by subdividing it into little pieces called elements. Although finite elements are geometrically more versatile than finite differences and finite volumes, their algorithms are more sophisticated and require special care of implementation.

Once time and space are discretized, we obtain a system of algebraic equations. The solution of this system characterizes the evolution of all continuous fields, both in space and time. Depending of the required level of accuracy, the solution procedure can become a challenging and computationally expensive task. Consequently, we can select between various useful simplifications: In the real world, the domain of interest is three dimensional. However, we can often extract useful information about the biological system by approximating it as one or two dimensional. Another simplification could be a particular symmetry of the system. Typical examples are cylindrical symmetries, which can be approximated by one-dimensional formulation referred to as axisymmetric.

Discrete models are typically represented through ordinary differential equations. These equations do not need to be solved over the entire domain; they are solved locally to predict the evolution of each individual entity in time. Because only the time needs to be partitioned into small intervals, and not the space, it is common to use the finite difference method. Although discrete models are conceptually simpler than continuum models, they are computationally more expensive because every single entity has to be represented through its own equation. When dealing with entire cell populations, their computational cost becomes overwhelming.

\section{GUIDELINES}

From the above discussion on the basic approaches toward systems-based mathematical representations, and to establish a framework for a guided discussion of the most recent wound healing models, we propose a few useful guidelines for effective computational modeling of wound healing.

\section{Simplified One-Dimensional and Axisymmetric Models Can Provide Quick Insight}

One-dimensional and axisymmetric models provide a quick first insight into the interplay of individual signaling pathways during wound healing. They are of great value for quickly testing therapeutic hypotheses. Because of their conceptual simplicity, one-dimensional and axisymmetric models should be viewed as a validated and well-calibrated starting point for further model refinement. For detailed analyses that focus on the spatiotemporal interaction of signaling pathways in wound healing, however, we recommend using two- or three-dimensional models.

\section{Discrete Models Can Answer Fundamental Questions at the Molecular and Cellular Levels}

We recommend using discrete models to study the biochemistry of individual cells. Discrete models are typically based on simple relations and straightforward to implement. Because they are computationally expensive, however, they are only feasible for answering fundamental scientific questions at small scales. We recommend fully avoiding discrete models when diffusion or mechanical cues play an important role.

\section{Continuum Models Can Answer Fundamental Questions at the Tissue, Organ, and System Levels}

We recommend using continuum models to study the interaction of biochemical and biomechanical fields. Continuum models are computationally efficient but require special care when translating the collective action of individual cells to a continuous field. When embedded in efficient finite element solvers, continuum models allow for a patient-specific analysis based on individualized, realistic three-dimensional geometries. Continuum models are ideal to answer translational clinical questions at larger scales.

\section{Multiple-Field Models Provide Insight Into the Cross-Talk Between Chemistry, Biology, and Mechanics}

Although models focusing on a single individual aspect of wound healing can provide specific insight, holistic models are currently becoming the gold standard in wound healing simulations. By contrast to single-field models, holistic multifield models can reliably represent the cross-signaling 
networks during wound healing. When creating a multifield model, we recommend including a representation of at least five interacting fields: (i) fibroblast population; (ii) endothelial cell population and/or nutrient concentration; (iii) indicator of ECM restoration such as collagen content; (iv) chemoattractant concentration; and (v) inflammatory cells population. This baseline five-field model can, of course, be further refined if specific aspects of the healing process are of interest.

\section{SYSTEMS-BASED MATHEMATICAL MODELS OF WOUND HEALING}

Over the past two decades, mathematical and computational modeling have advanced as key players in the quest for understanding the complex multiscale phenomena involved in wound healing. Ideally, once calibrated and validated, these models can serve as tools to quantify the impact of selected perturbations to the baseline system and, ultimately, to predict the effect of different therapeutic treatment options.

In an effort to gain basic scientific insight into the process of wound healing, researchers in the early ' 90 s started to develop mathematical models of the repair response of skin. Sherrat and Murray are often considered to be the fathers of modern mathematical modeling of wound healing (42). Since their first model was published more than two decades ago, a multitude of modifications, improvements, and enhancements have been proposed. The characteristics, the focus, and the simplifications of each model are different, based on the fundamental question it seeks to answer. This makes it challenging to define a unique benchmark for a scientist or clinician who would like to make use of the existing models or propose a new one.

It is becoming clear, however, that the current trend in computational systems biology converges toward multiscale, multifield modeling. In the time domain, most analyses focus on shorttime scales, mainly on the inflammatory and proliferative phases, and only a few more recent models integrate larger times scales including the remodeling period (43). In the space domain, modelers are now giving priority to multiscale integration, from a basic mathematical representation of the cell to fully three-dimensional models of the wound site.

Here, we suggest a classification of existing models based on the aspect of wound healing they seek to address and on the mathematical tools they use to derive their governing equations, see Table 1. In this classification, the three major focus areas are re-epithelialization and cell migration; angiogenesis; and wound contraction and collagen deposition. The four degrees of modeling complexity are one-dimensional or axisymmetric continuum models; two-dimensional continuum models; two- or three-dimensional discrete models; and twodimensional hybrid discrete/continuum approaches.

We hope this classification alongside the guidelines discussed above will provide new computational scientists and clinicians with the necessary framework to make use of the existing models or to create improved versions attending to the specific question they want to answer.

To better illustrate which modeling approaches have been used and how they have enhanced the understanding of wound healing from the perspective of systems biology, the following subsections present, for each major focus area defined in Table 1, the modeling and solution methods that have proven to be more effective, followed by an example of how computational simulations have tested virtual hypotheses by altering the baseline model and analyzing the outcome of the computations.

\section{Re-epithelialization Creates Propagation Patterns of Constant Velocity Waves}

The simplest mathematical models in wound healing focus on re-epithelialization and cell migration. These models typically characterize a cell population over a domain in terms of point-wise cell densities rather than considering each individual cell. Fisher's equation is the preferred representation of the population behavior. It consists of a diffusion term to capture cell migration and a logistic term to capture cell proliferation (39). This abstraction is well suited for the study of re-epithelialization and is also considered useful for fibroblast and endothelial cell migration in wound healing $(39,44)$. During re-epithelialization, keratinocytes remain in stretch contact with one another, maintaining tissue continuity throughout the epidermis. A continuum assumption is therefore well justified. The solution to Fisher's equation is a wave propagation pattern in which the advancing front of cells moves with an almost constant velocity (44). Although the model is conceptually two dimensional $(45,46)$, most numerical implementations are based on the additional assumption of axisymmetry to further simplify the modeling. A more sophisticated alternative to these continuum models is the discrete model, which represents each individual cell explicitly (47). Discrete models typically describe the motion of each cell as a reinforced random walk in the direction of a stimulus and regulate cell division through an internal clock (48).

Table 1. Systematic classification of mathematical models for wound healing based on model complexity and focus area

\begin{tabular}{lccc} 
& & \multicolumn{2}{c}{ Reference citation number } \\
\cline { 2 - 4 } Complexity/focus & $\begin{array}{c}\text { Re-epithelialization and cell } \\
\text { migration }\end{array}$ & Angiogenesis & $\begin{array}{c}\text { Wound contraction and } \\
\text { collagen deposition }\end{array}$ \\
\hline One-dimensional continuum & 39,44 & $34,41,51,52,53,54,59,60$, & $43,66,67,69,75,77$ \\
Two-dimensional continuum & & 64,65 & 71 \\
Two-dimensional/three-dimensional discrete & 45,46 & $25,49,55,56$ \\
Two-dimensional hybrid discrete/continuum & 47,48 & 57 & $68,70,76$ \\
\hline
\end{tabular}


Re-epithelialization Can Be Manipulated by Altering TGF- $\beta 1$ Kinetics

During wound healing, keratinocytes, initially located at the edge of the lesion, crawl over the lesion to restore the protective barrier. Although cell-cell cross-talk is key to this process, external signaling strongly influences the behavior of the leading edge. It overwrites the default program to initiate horizontal growth of skin, temporally bypassing the upward motion of keratinocytes from the basal membrane to the stratum corneum. In this early response of keratinocytes, TGF- $\beta 1$ has been recognized as a crucial signaling pathway controlling two major events: it increases keratinocyte migration and decreases keratinocyte proliferation. A balanced action of TGF- $\beta 1$ is therefore critical to avoid pathological re-epithelialization. With the help of agent-based computational models, Sun et al. (49) explored the role of TGF- $\beta 1$ in epidermal wound healing and identified spatiotemporal sequences of events in normal and pathological wound healing. In their baseline model, increased concentration of TGF- $\beta 1$ at the edge of the wound induced a population of keratinocytes to migrate inward and blocked their proliferation. As the leading edge moves into the lesion, the adjacent population of keratinocytes was exposed to normal levels of TGF- $\beta 1$ but to an increased presence of other growth factors that stimulate cell proliferation. Normal re-epithelialization can thus be viewed as a nonproliferative, highly motile leading edge followed by a proliferative population of keratinocytes. In these computational experiments, disrupting the balance of TGF- $\beta 1$ created either chronic wounds or hypertrophic scars (49).

\section{Angiogenesis Creates Fractal Patterns of Vascular Networks}

The aspect of wound healing that has received the most attention in modeling is angiogenesis. The reason for the disproportional interest in new vessel formation is that it is not only crucial to wound healing but also to tumor growth. The basic assumptions of angiogenesis models are valid for both circumstances,

a

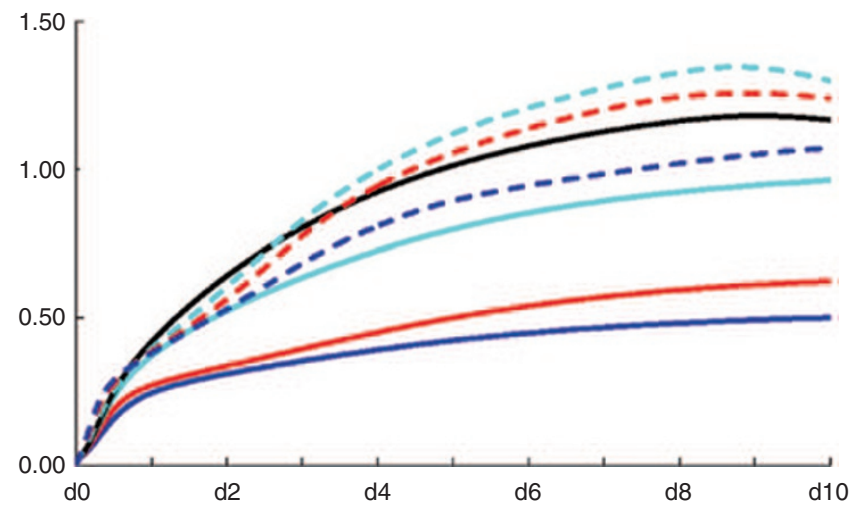

and combined efforts between these two scientific communities have markedly pushed the frontiers in mathematical modeling of angiogenesis within the past decade $(40,50)$. Researchers in wound healing and tumor growth have collectively used various approaches to study the creation of new vasculature, and some of their most remarkable models are extremely elaborate. They include most features of the repair sequence: inflammatory cells, one or more chemoattractants, fibroblasts, and the ECM $(41,51)$. In continuum models, new vasculature is represented as the density of endothelial cells in a point-wise fashion or as a combination of capillary tip and sprout densities (52). A partial differential equation dictates the evolution of the endothelial cell density field, in analogy to Fisher's equation, but with additional coupling terms between the endothelial cells and the other fields in their immediate environment $(53,54)$. In discrete models, fractal-like approaches or cellular automata have been proposed to account for the contact between individual endothelial cells as the capillary tubes branch out $(55,56)$. Hybrid models have also successfully reproduced the angiogenesis mechanism (57). The greatest value of angiogenesis models, continuous or discrete, is that they allow us to explore the effects of altered nutrient and oxygen supplies on the healing process.

\section{Angiogenesis Can Be Manipulated by Altering Hyperbaric Oxygen Levels}

During wound healing, elevated metabolic needs in the proliferative phase increase oxygen demand. In fact, the lack of oxygen or hypoxia is prevalent in the wound during the inflammatory phase and is an important cue for macrophages to release cytokines that recruit other cell types for the subsequent phases (58-60). If hypoxia is severe, for example in chronic wounds, the tissue is incapable of creating new vasculature, and the healing process is significantly impaired (61). Thus, oxygen therapy has been proposed to accelerate the healing process (62). However, excess of oxygen can also be harmful, because it can lead to

b

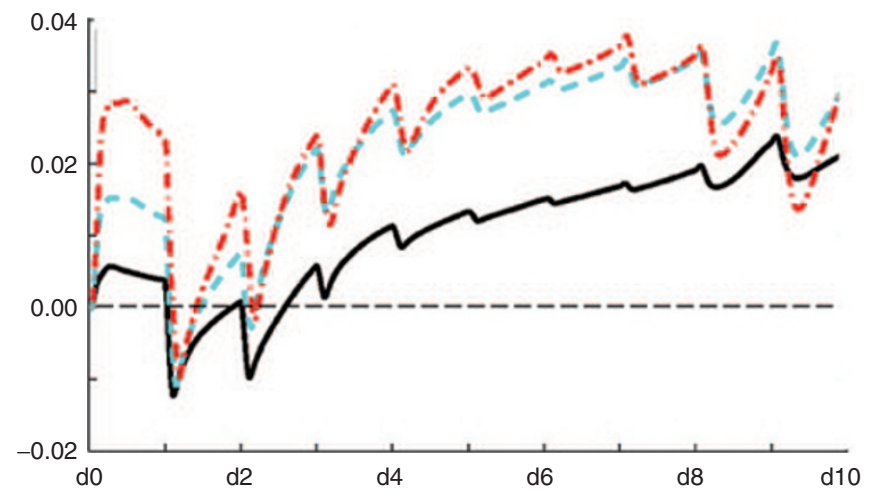

Figure 2. Angiogenesis can be manipulated by altering hyperbaric oxygen levels. (a) The baseline system (solid black line) shows endothelial cell density for oxygen levels in the absence of hyperbaric oxygen therapy during a period of $10 \mathrm{~d}$. Hyperoxic conditions can enhance healing; however, excess of oxygen can be harmful (dashed cyan line: $\mathrm{O}_{2}=2.00 \%$; dashed red line: $\mathrm{O}_{2}=3.00 \%$; and dashed blue line: $\mathrm{O}_{2}=4.00 \%$ ). Hypoxic conditions impair the healing response (solid cyan line: $\mathrm{O}_{2}=0.75 \%$; solid red line: $\mathrm{O}_{2}=0.50 \%$; and solid blue line: $\mathrm{O}_{2}=0.25 \%$ ). (b) Hyperbaric oxygen therapy is not constant over the healing period; rather, oxygen is administered daily in short sessions. A common therapy consists of $\mathrm{O}_{2}=2.00 \%, 90 \mathrm{~min} / \mathrm{d}$ (solid black line) leading to increased endothelial cell density over the same 10-d period. Computational simulations can predict alternative therapies (dashed cyan line: $\mathrm{O}_{2}=3.00 \%$, $90 \mathrm{~min} / \mathrm{d}$; and dashed red line: $\mathrm{O}_{2}=4.00 \%, 90 \mathrm{~min} / \mathrm{d}$ ). Adapted with permission from ref. 65 . Copyright (2008) National Academy of Sciences, USA. 
intoxication (63). Hypoxia is an essential cue during the inflammatory phase and is linked indirectly to fibroblast recruitment (62). The interplay of oxygen and cellular recruitment has successfully been studied with the aid of computational models (64).

For example, Schugart et al. proposed a model of wound healing in which oxygen tension across the wound is considered as an additional variable (65). Their baseline model reproduced a normal healing response throughout a period of $10 \mathrm{~d}$. Then, different degrees of hypoxia and hyperpoxia were simulated. Their simulations revealed that severe hypoxia cannot sustain angiogenesis and that extreme hyperpoxia reduces the proliferation of endothelial cells (see Figure 2, top). Finally, they studied different hyperbaric oxygen therapies and concluded that $90 \mathrm{~min}$ of hyperbaric oxygen per day enhances the healing process (see Figure 2, bottom).

Wound Contraction by Myofibroblasts Creates Tension in the ECM Similar to angiogenesis, collagen deposition and wound contraction have mainly been studied holistically $(66,67)$. Unique features of their mathematical models are the consideration of fibroblasts and myofibroblasts $(68,69)$. Fully discrete models are not appealing to represent the ECM, and therefore modelers have turned to either hybrid or fully continuous frameworks. In the former, the chemical species and the ECM are modeled as continuous fields, whereas the cells are modeled discretely as individual entities (70). The major focus of hybrid discrete/continuum models has been on collagen deposition. If we decide to also represent cell populations as continuous fields, we can select plain continuum models and more advanced mechanical theories to answer questions such as those regarding myofibroblasts-based active wound contraction (71).

\section{Collagen Deposition Can Be Manipulated by Altering TGF- $\beta$ Kinetics}

Although the biochemical aspects of wound healing have received significant attention, mathematical modeling of the mechanical aspects of wound healing remains largely unexplored. The role of mechanical cues is currently gaining a
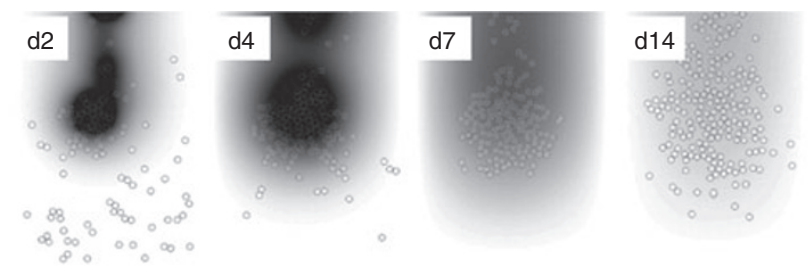

$\mathrm{d} 2$
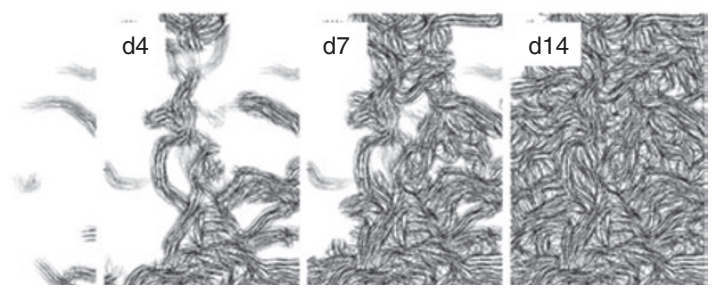

b
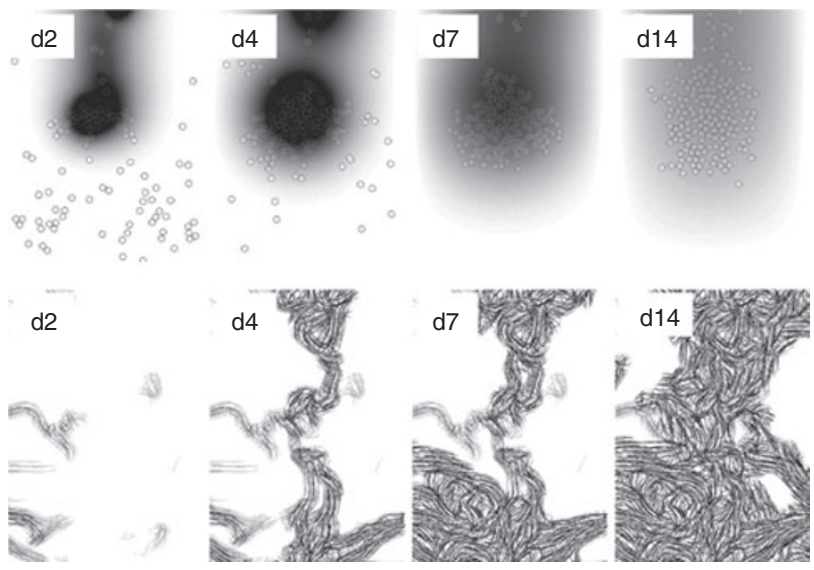

Figure 3. Collagen deposition can be manipulated by altering transforming growth factor- $\beta$ (TGF- $\beta$ ) kinetics. Two-dimensional hybrid discrete/continuum simulation of wound healing with TGF- $\beta$ concentration color-coded in gray, top; fibroblasts displayed as discrete cells, top; and collagen fibers displayed through local fiber orientation maps, bottom. (a) Normal TGF- $\beta$ diffusion initiates progressive collagen deposition at days $2,4,7$, and 14, whereas (b) reduced TGF- $\beta$ diffusion generates localized fibroblast concentrations and reduced collagen deposition. Adapted with permission from Figures 7 and 9 in ref. 76.
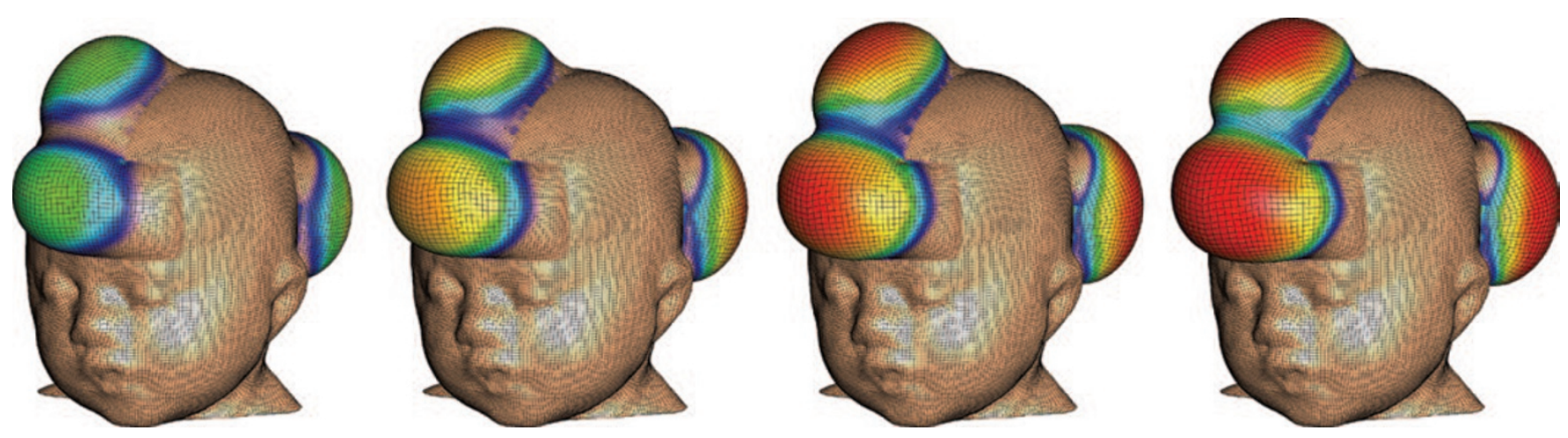

Figure 4. Collagen deposition and mitosis can be manipulated by altering stretch levels. Tissue expansion uses the concept of systematic overstretch to create extra skin for defect repair in reconstructive surgery. Gradually increasing the mechanical stretch over a period of $12 \mathrm{wk}$, from left to right, triggers a gradual increase in skin area, from blue to red. In the red regions, skin has more than doubled its initial size. From ref. 84. 
importance, however, because recent experimental data suggest that releasing mechanical stresses in the wound may shorten the inflammatory phase and reduce scarring $(8,72)$. Mechanical stress is transmitted across the ECM and directly affects fibroblast behavior $(73,74)$. For example, the local environment of a fibroblast can induce its transformation into a myofibroblast (75). Myofibroblasts are responsible for contracting the tissue and bringing the edges of the wound together (69). Although there are a few models that have incorporated wound contraction by myofibroblasts, these models have not yet been used to optimize the mechanical environment and enhance therapeutic outcomes. Nonetheless, because pathologic scarring is a major concern in wound healing, researchers have focused on modeling the flow of chemical information, which regulates collagen deposition and creation of the new ECM (75).

For example, Cumming et al. studied the response of fibroblasts to TGF- $\beta$, a cytokine released by macrophages during inflammation, throughout a period of $14 \mathrm{~d}$ (76). Using a predictive mathematical model, they were able to show that altered TGF- $\beta$ kinetics in the wound have a significant impact on the healing process. They found that in their baseline model, fibroblasts tend to cluster around the zones with the highest concentration of chemoattractants, where they gradually replace the fibrin clot with collagen as they reach the damaged region (see Figure 3, left). According to the simulations, reduced TGF- $\beta$ diffusion causes a clustering of fibroblasts, reduced collagen synthesis, and significantly altered healing kinetics (see Figure 3, right). Another remarkable example of the use of agent-based models is the study conducted by $\mathrm{Mi}$ et al. (77), which focused on TGF- $\beta 1$, a specific isoform of TGF- $\beta$. Altered kinetics of this chemokine are an important factor in diabetic ulcer pathology. According to their simulations, increased tumor necrosis factor- $\alpha$ and decreased TGF$\beta 1$ lead to an impaired healing response of diabetic patients. The model further showed that altering these chemoattractants increased the concentration of TGF- $\beta 1$, increased collagen deposition, reduced concentration of tumor necrosis factor- $\alpha$, and reduced necrosis.

\section{Collagen Deposition and Mitosis Can Be Manipulated by Altering Stretch Levels}

Despite intense research over the past two decades, most existing models for wound healing are still one or two dimensional and focus on the acute rather than on the chronic response (see Table 1). The majority of these models use finite difference or finite volume methods to discretize their governing equations in space, limiting the geometries to idealized settings. An elegant way to incorporate realistic three-dimensional geometries of the wound and skin anatomy is finite element modeling. Recent trends in computational biology focus on predicting chronic soft-tissue adaptation using mechanistic finite element models $(78,79)$. In an attempt to quantify how elevated mechanical stretch can alter collagen deposition and fibroblast mitosis, several groups have recently modeled chronic skin growth in response to changes in the mechanical environment $(80,81)$. These models have immediate clinical applications in skin expansion in plastic and reconstructive surgery. Predicting stress, strain, and skin area gain, skin growth models have the potential to enhance treatment for patients with birth defects, burn injuries, or breast tumor removal (82).

For example, Zöllner et al. simulated skin expansion in pediatric forehead reconstruction of a 1-y-old girl throughout a period of $12 \mathrm{wk}$ (83). Their model incorporated a thermomechanically consistent representation of the dermis and a phenomenological scalar field that quantified the amount of newly grown skin (82). A conceptually simple and elegant abstraction of the mechanotransduction pathways and the corresponding cellular response defined the evolution of this scalar field in terms of mechanical stimuli such as tissue stretch $(83,84)$ (see Figure 4). Refining this framework to incorporate the true mechanotransduction pathways during mechanical overstretch or during wound healing appears to be a next logical step toward predicting and improving of wound healing therapies in realistic three-dimensional geometries of pediatric patients.

\section{PERSPECTIVE}

Systems-based mathematical modeling of wound healing has achieved remarkable sophistication and is on its way to becoming an irreplaceable tool in personalized medicine. The first mathematical models of wound healing were proposed two decades ago and considered only specific aspects of the healing process. Current models include most of the key components that interact throughout the repair sequence. They have opened the floor to advanced hypothesis testing and enhanced wound management therapies. The state of the art in computational modeling of wound healing is the temporal and spatial integration of different cell types, chemoattractants, nutrients, and the ECM, interacting jointly to restore tissue integrity. Current trends in computational modeling indicate that this knowledge gained on the cellular level should be integrated in holistic multiscale multifield continuum models through a bottom-up approach. The ultimate goal would be to create high-resolution system-level models with parameters calibrated at their generative level of resolution. Rather than fitting physiologically meaningless phenomenological parameters to system-level measurements, which has been the standard for the past decades, all parameters would then have a clear physiological interpretation.

Greatest attention has been paid to the biochemical features of healing such as diffusion of chemoattractants and oxygen tension. The first models have now advanced far enough to reliably predict how systematic manipulations of baseline parameters in chemical signaling can change the healing process. The biomechanical features of healing are currently receiving increasing attention. Mechanical models focus primarily on collagen deposition and active wound contraction. Yet, to date, it still remains unclear how exactly these mechanisms are regulated when the injured skin tries to recover its homeostatic equilibrium state.

One of the major challenges in the mathematical and computational modeling of wound healing is the consideration of complex, physiological geometries in two and three dimensions. 
Current models have been tested primarily in axisymmetric conditions, which limits their application to a clinical setting and reduces their translational potential. Another current road block is the incorporation of a detailed mechanical representation of skin with temporally, spatially, and directionally varying material properties. Fortunately, recent advances in molecular biology, mechanotransduction, soft-tissue mechanics, and computational modeling of soft tissues might soon allow us to bridge these gaps. The first patient-specific computational model of pediatric wound healing is likely to appear within this decade, and it will constitute a major breakthrough in the progress of systems biology toward a better care for the pediatric patient.

\section{STATEMENT OF FINANCIAL SUPPORT}

This work was supported by the Consejo Nacional de Ciencia y Tecnologia (CONACYT) Fellowship and the Stanford Graduate Fellowship to A.B.-T.; by the National Science Foundation CAREER award CMMI-0952021 and INSPIRE award 1233054; and by the National Institutes of Health grant U54 GM072970 to E.K.

Disclosure: The authors declare that they have no conflict of interest and no relationships with industry relevant to this work.

\section{REFERENCES}

1. Diegelmann RF, Evans MC. Wound healing: an overview of acute, fibrotic and delayed healing. Front Biosci 2004;9:283-9.

2. Gurtner GC, Werner S, Barrandon Y, Longaker MT. Wound repair and regeneration. Nature 2008;453:314-21.

3. Colwell AS, Longaker MT, Lorenz HP. Fetal wound healing. Front Biosci 2003;8:s1240-8.

4. Mutsaers SE, Bishop JE, McGrouther G, Laurent GJ. Mechanisms of tissue repair: from wound healing to fibrosis. Int J Biochem Cell Biol 1997;29:5-17.

5. Verhaegen PD, van Zuijlen PP, Pennings NM, et al. Differences in collagen architecture between keloid, hypertrophic scar, normotrophic scar, and normal skin: an objective histopathological analysis. Wound Repair Regen 2009;17:649-56.

6. Brown BC, McKenna SP, Siddhi K, McGrouther DA, Bayat A. The hidden cost of skin scars: quality of life after skin scarring. J Plast Reconstr Aesthet Surg 2008;61:1049-58.

7. Bayat A, McGrouther DA, Ferguson MW. Skin scarring. BMJ 2003; 326:88-92.

8. Gurtner GC, Dauskardt RH, Wong VW, et al. Improving cutaneous scar formation by controlling the mechanical environment: large animal and phase I studies. Ann Surg 2011;254:217-25.

9. Christley S, Lee B, Dai X, Nie Q. Integrative multicellular biological modeling: a case study of 3D epidermal development using GPU algorithms. BMC Syst Biol 2010;4:107.

10. Vodovotz Y, Csete M, Bartels J, Chang S, An G. Translational systems biology of inflammation. PLoS Comput Biol 2008;4:e1000014.

11. Kitano H. Computational systems biology. Nature 2002;420:206-10.

12. Vodovotz Y. Translational systems biology of inflammation and healing. Wound Repair Regen 2010;18:3-7.

13. Martin P. Wound healing-aiming for perfect skin regeneration. Science 1997;276:75-81.

14. Velnar T, Bailey T, Smrkolj V. The wound healing process: an overview of the cellular and molecular mechanisms. J Int Med Res 2009;37: 1528-42.

15. Olutoye OO, Zhu X, Cass DL, Smith CW. Neutrophil recruitment by fetal porcine endothelial cells: implications in scarless fetal wound healing. Pediatr Res 2005;58:1290-4.

16. Hunter PJ, Borg TK. Integration from proteins to organs: the Physiome Project. Nat Rev Mol Cell Biol 2003;4:237-43.

17. Qutub AA, Mac Gabhann F, Karagiannis ED, Vempati P, Popel AS. Multiscale models of angiogenesis. IEEE Eng Med Biol Mag 2009;28: $14-31$.
18. Southern J, Pitt-Francis J, Whiteley J, et al. Multi-scale computational modelling in biology and physiology. Prog Biophys Mol Biol 2008;96:60-89.

19. Painter KJ. Continuous models for cell migration in tissues and applications to cell sorting via differential chemotaxis. Bull Math Biol 2009;71:1117-47.

20. Postlethwaite AE, Keski-Oja J, Moses HL, Kang AH. Stimulation of the chemotactic migration of human fibroblasts by transforming growth factor beta. J Exp Med 1987;165:251-6.

21. Tranquillo RT, Lauffenburger DA. Stochastic model of leukocyte chemosensory movement. J Math Biol 1987;25:229-62.

22. Steenfos HH. Growth factors and wound healing. Scand J Plast Reconstr Surg Hand Surg 1994;28:95-105.

23. Insall RH. Understanding eukaryotic chemotaxis: a pseudopod-centred view. Nat Rev Mol Cell Biol 2010;11:453-8.

24. Wadhams GH, Armitage JP. Making sense of it all: bacterial chemotaxis. Nat Rev Mol Cell Biol 2004;5:1024-37.

25. Stokes CL, Lauffenburger DA. Analysis of the roles of microvessel endothelial cell random motility and chemotaxis in angiogenesis. J Theor Biol 1991;152:377-403.

26. Wong VW, Levi K, Akaishi S, Schultz G, Dauskardt RH. Scar zones: regionspecific differences in skin tension may determine incisional scar formation. Plast Reconstr Surg 2012;129:1272-6.

27. Ogawa R. Keloid and hypertrophic scarring may result from a mechanoreceptor or mechanosensitive nociceptor disorder. Med Hypotheses 2008;71:493-500.

28. Wong VW, Akaishi S, Longaker MT, Gurtner GC. Pushing back: wound mechanotransduction in repair and regeneration. J Invest Dermatol 2011;131:2186-96.

29. Chiquet M, Gelman L, Lutz R, Maier S. From mechanotransduction to extracellular matrix gene expression in fibroblasts. Biochim Biophys Acta 2009;1793:911-20.

30. Paterno J, Vial IN, Wong VW, et al. Akt-mediated mechanotransduction in murine fibroblasts during hypertrophic scar formation. Wound Repair Regen 2011;19:49-58.

31. Wong VW, Rustad KC, Akaishi S, et al. Focal adhesion kinase links mechanical force to skin fibrosis via inflammatory signaling. Nat Med 2012;18:148-52.

32. Aarabi S, Bhatt KA, Shi Y, et al. Mechanical load initiates hypertrophic scar formation through decreased cellular apoptosis. FASEB J 2007:21:3250-61.

33. Simpson CL, Patel DM, Green KJ. Deconstructing the skin: cytoarchitectural determinants of epidermal morphogenesis. Nat Rev Mol Cell Biol 2011;12:565-80.

34. Levine HA, Sleeman BD, Nilsen-Hamilton M. Mathematical modeling of the onset of capillary formation initiating angiogenesis. J Math Biol 2001;42:195-238.

35. Herbert SP, Stainier DY. Molecular control of endothelial cell behaviour during blood vessel morphogenesis. Nat Rev Mol Cell Biol 2011;12:551-64.

36. Tong P, Fung YC. The stress-strain relationship for the skin. J Biomech 1976;9:649-57.

37. Lanir Y. Constitutive equations for fibrous connective tissues. J Biomech 1983;16:1-12.

38. Flynn C, Taberner A, Nielsen P. Mechanical characterisation of in vivohuman skin using a 3D force-sensitive micro-robot and finite element analysis. Biomech Model Mechanobiol 2011;10:27-38.

39. Maini PK, McElwain DL, Leavesley DI. Traveling wave model to interpret a wound-healing cell migration assay for human peritoneal mesothelial cells. Tissue Eng 2004;10:475-82.

40. Chaplain MA. Mathematical modelling of angiogenesis. J Neurooncol 2000;50:37-51.

41. Pettet GJ, Byrne HM, McElwain DL, Norbury J. A model of wound-healing angiogenesis in soft tissue. Math Biosci 1996;136:35-63.

42. Sherratt JA, Murray JD. Models of epidermal wound healing. Proc Biol Sci 1990;241:29-36.

43. Cobbold CA, Sherratt JA. Mathematical modelling of nitric oxide activity in wound healing can explain keloid and hypertrophic scarring. J Theor Biol 2000;204:257-88.

44. Sherratt JA, Murray JD. Mathematical analysis of a basic model for epidermal wound healing. J Math Biol 1991;29:389-404. 
45. Haugh JM. Deterministic model of dermal wound invasion incorporating receptor-mediated signal transduction and spatial gradient sensing. Biophys J 2006;90:2297-308.

46. Javierre E, Vermolen FJ, Vuik C, van der Zwaag S. A mathematical analysis of physiological and morphological aspects of wound closure. J Math Biol 2009;59:605-30.

47. Cai AQ, Landman KA, Hughes BD. Multi-scale modeling of a woundhealing cell migration assay. J Theor Biol 2007;245:576-94.

48. Callaghan T, Khain E, Sander L. A stochastic model for wound healing. J Stat Phys 2006;122:909-24.

49. Sun T, Adra S, Smallwood R, Holcombe M, MacNeil S. Exploring hypotheses of the actions of TGF-beta1 in epidermal wound healing using a $3 \mathrm{D}$ computational multiscale model of the human epidermis. PLoS ONE 2009;4:e8515.

50. Peirce SM. Computational and mathematical modeling of angiogenesis. Microcirculation 2008;15:739-51.

51. Xue C, Friedman A, Sen CK. A mathematical model of ischemic cutaneous wounds. Proc Natl Acad Sci USA 2009;106:16782-7.

52. Gaffney EA, Pugh K, Maini PK, Arnold F. Investigating a simple model of cutaneous wound healing angiogenesis. J Math Biol 2002;45:337-74.

53. Anderson A. A mathematical model for capillary network formation in the absence of endothelial cell proliferation. Appl Math Lett 1998;11:109-14.

54. Byrne HM, Chaplain MAJ, Evans DL, Hopkinson I. Mathematical modelling of angiogenesis in wound healing: Comparison of theory and experiment. J Theor Med 2000;2:175-97.

55. Peirce SM, Van Gieson EJ, Skalak TC. Multicellular simulation predicts microvascular patterning and in silicotissue assembly. FASEB J 2004;18:731-3.

56. Sleeman BD, Levine HA. Partial differential equations of chemotaxis and angiogenesis. Math Meth Appl Sci 2001;24:405-26.

57. MildeF,BergdorfM,KoumoutsakosP.Ahybrid modelforthree-dimensional simulations of sprouting angiogenesis. Biophys J 2008;95:3146-60.

58. Knighton DR, Hunt TK, Scheuenstuhl H, Halliday BJ, Werb Z, Banda MJ. Oxygen tension regulates the expression of angiogenesis factor by macrophages. Science 1983;221:1283-5.

59. Maggelakis SA. A mathematical model of tissue replacement during epidermal wound healing. Appl Math Model 2003;27:189-96.

60. Owen MR, Byrne HM, Lewis CE. Mathematical modelling of the use of macrophages as vehicles for drug delivery to hypoxic tumour sites. J Theor Biol 2004;226:377-91.

61. Sen CK. Wound healing essentials: let there be oxygen. Wound Repair Regen 2009;17:1-18.

62. Gordillo GM, Sen CK. Revisiting the essential role of oxygen in wound healing. Am J Surg 2003;186:259-63.

63. Tibbles PM, Edelsberg JS. Hyperbaric-oxygen therapy. N Engl J Med 1996;334:1642-8.

64. Croll TI, Gentz S, Mueller K, et al. Modelling oxygen diffusion and cell growth in a porous, vascularising scaffold for soft tissue engineering applications. Chem Eng Sci 2005;60:4924-34.

65. Schugart RC, Friedman A, Zhao R, Sen CK. Wound angiogenesis as a function of tissue oxygen tension: a mathematical model. Proc Natl Acad Sci USA 2008;105:2628-33.

66. Olsen L, Sherratt JA, Maini PK. A mathematical model for fibro-proliferative wound healing disorders. Bull Math Biol 1996;58:787-808.
67. Olsen L, Sherratt JA, Maini PK. A mechanochemical model for adult dermal wound contraction and the permanence of the contracted tissue displacement profile. J Theor Biol 1995;177:113-28.

68. Dallon JC, Sherratt JA, Maini PK. Modeling the effects of transforming growth factor-beta on extracellular matrix alignment in dermal wound repair. Wound Repair Regen 2001;9:278-86.

69. Tranquillo RT, Murray JD. Continuum model of fibroblast-driven wound contraction: inflammation-mediation. Biomech Model Mechanobiol 2007;158:361-71.

70. McDougall S, Dallon J, Sherratt J, Maini P. Fibroblast migration and collagen deposition during dermal wound healing: mathematical modelling and clinical implications. Philos Transact A Math Phys Eng Sci 2006;364:1385-405.

71. Vermolen FJ, Javierre E. Computer simulations from a finite-element model for wound contraction and closure. J Tissue Viability 2010;19:43-53.

72. Yagmur C, Akaishi S, Ogawa R, Guneren E. Mechanical receptor-related mechanisms in scar management: a review and hypothesis. Plast Reconstr Surg 2010;126:426-34.

73. Prajapati RT, Eastwood M, Brown RA. Duration and orientation of mechanical loads determine fibroblast cyto-mechanical activation: monitored by protease release. Wound Repair Regen 2000;8:238-46.

74. Prajapati RT, Chavally-Mis B, Herbage D, Eastwood M, Brown RA. Mechanical loading regulates protease production by fibroblasts in three-dimensional collagen substrates. Wound Repair Regen 2000;8: 226-37.

75. Murphy KE, McCue SW, McElwain DL. Clinical strategies for the alleviation of contractures from a predictive mathematical model of dermal repair. Wound Repair Regen 2012;20:194-202.

76. Cumming BD, McElwain DL, Upton Z. A mathematical model of wound healing and subsequent scarring. J R Soc Interface 2010;7: 19-34.

77. Mi Q, Rivière B, Clermont G, Steed DL, Vodovotz Y. Agent-based model of inflammation and wound healing: insights into diabetic foot ulcer pathology and the role of transforming growth factor-beta1. Wound Repair Regen 2007;15:671-82.

78. Ambrosi D, Ateshian GA, Arruda EM, et al. Perspectives on biological growth and remodeling. J Mech Phys Solids 2011;59:863-83.

79. Menzel A, Kuhl E. Frontiers in growth and remodeling. Mech Res Commun 2012;42:1-14.

80. Socci L, Pennati G, Gervaso F, Vena P. An axisymmetric computational model of skin expansion and growth. Biomech Model Mechanobiol 2007;6: 177-88.

81. Buganza Tepole A, Gosain A, Kuhl E. Stretching skin: the physiological limit and beyond. Int J Non-Linear Mech 2012;47: 938-49.

82. Buganza Tepole A, Ploch CJ, Wong J, Gosain AK, Kuhl E. Growing skin: a computational model for skin expansion in reconstructive surgery. J Mech Phys Solids 2011;59:2177-90.

83. Zöllner AM, Buganza Tepole A, Gosain AK, Kuhl E. Growing skin: tissue expansion in pediatric forehead reconstruction. Biomech Model Mechanobiol 2012;11:855-67.

84. Zöllner AM, Buganza Tepole A, Kuhl E. On the biomechanics and mechanobiology of growing skin. J Theor Biol 2012;297:166-75. 